\title{
Dietary fructo-oligosaccharides in healthy adults do not negatively affect faecal cytotoxicity: a randomised, double-blind, placebo-controlled crossover trial
}

\author{
Petra A. M. J. Scholtens ${ }^{1}{ }^{*}$, Martine S. Alles ${ }^{1}$, Linette E. M. Willemsen ${ }^{2}$, Claudia van den Braak ${ }^{2}$, \\ Jacques G. Bindels ${ }^{1}$, Günther Boehm ${ }^{1}$ and Mirjam J. A. P. Govers ${ }^{2}$ \\ ${ }^{1}$ Department of Baby Food Research, Numico Research BV, Wageningen, The Netherlands \\ ${ }^{2}$ Department of Biomedical Research, Numico Research BV, Wageningen, The Netherlands
}

(Received 20 July 2005 - Revised 30 January 2006 - Accepted 30 January 2006)

Fructo-oligosaccharides (FOS) are widely used in commercial food products. Most studies on FOS concern the health benefits, but some negative effects were recently reported concerning the faecal cytotoxicity and excretion of mucin-type oligosaccharides in combination with a Ca-restricted diet. The present study was performed to investigate whether these effects of FOS are observed in adults consuming a regular diet unrestricted in $\mathrm{Ca}$. The study was a randomised, double-blind, placebo-controlled crossover trial, involving eleven healthy adults, who consumed 25-30 g FOS or maltodextrin (control) in a random order for 2 weeks in addition to their regular diet. Stools were collected for analysis of pH and SCFA (as markers of fermentation), for the assessment of faecal water cytotoxicity, and for the analysis of alkaline phosphatase activity (as a marker of epithelial cell turnover) and $O$-linked oligosaccharides (to estimate the excretion of mucin-type oligosaccharides). FOS consumption significantly altered bacterial fermentation (increased percentage of acetate, decreased percentage of butyrate) and tended to decrease stool pH. Furthermore, FOS consumption resulted in a significantly higher stool frequency and in significantly more complaints of flatulence. No significant differences between the control and FOS period were observed in the mean cytotoxicity of faecal water (37.5 (SEM 6.9) \% v. 18.5 (SEM 6.9) $\% ; P=0.084$ ), in mean alkaline phosphatase activity (27.7 (SEM 2.9) v. 24.6 (SEM 3.2) U/g dry faeces; $P=0.496$ ) or in the mean excretion of mucin-type oligosaccharides (49.9 (SEM 4.0) v. 53.5 (SEM 4.3) $\mathrm{mg} / \mathrm{g}$ dry faeces; $P=0.553$ ). We conclude that dietary FOS in a dose up to $25-30 \mathrm{~g} / \mathrm{d}$ altered the bacterial fermentation pattern but did not affect faecal cytotoxicity or the faecal concentration of mucin-type oligosaccharides in human adults consuming a regular diet.

Fructo-oligosaccharides: Man: Cytotoxicity: Mucin: Alkaline phosphatase

Fructo-oligosaccharides (FOS) are plant fructans that naturally occur in, for example, chicory, onions, garlic, wheat and leeks. Fructans are non-digestible carbohydrates constructed of 2-60 monomer units (referred to as the degree of polymerisation). Fructans include both short-chain FOS (degree of polymerisation 2-7) and long-chain FOS (inulin; degree of polymerisation 2-60). The estimated mean daily intake of FOS and inulin from natural sources in a European diet is between 3.2 and $11.3 \mathrm{~g}$, with peak values up to $20 \mathrm{~g}$ (Van Loo, 1995).

FOS and inulin are widely used in commercial food products, both for their technological characteristics and for their health benefits. The health benefits of FOS and inulin can be attributed to their fermentation by the intestinal microbiota. Several studies suggest that FOS and inulin are involved in the selective stimulation of the growth of beneficial bacteria, such as bifidobacteria, in the gastrointestinal tract (reviewed by Kolida et al. 2002). Some of these studies have also shown a decrease in the number of pathogenic bacteria (Kolida et al. 2002).
Although most studies on the effect of FOS and inulin concern their health benefits, recent papers by the same group have reported that short-chain FOS (degree of polymerisation 2-7) in combination with a diet low in $\mathrm{Ca}$ were associated with an impairment in the resistance of rats to intestinal Salmonella infection (Bovee-Oudenhoven et al. 2003; Ten Bruggencate et al. 2003, 2004a). Prior to infection, an increased cytotoxicity of faecal water and an increased faecal excretion of mucin (estimated on the basis of faecal excretion of $O$-linked oligosaccharides) was observed in all three studies. In one of these studies (Ten Bruggencate et al. 2004a), it was shown that dietary Ca abolished the presumed detrimental effect of FOS. A recent study examined healthy males receiving a diet restricted in $\mathrm{Ca}$ (mean intake 298-315 $\mathrm{mg} \mathrm{Ca} / \mathrm{d}$ ) and supplemented with $20 \mathrm{~g}$ FOS or with $20 \mathrm{~g}$ sucrose for 2 weeks (Ten Bruggencate et al. 2004b). This study could not confirm the finding of the rat studies, with respect to the cytotoxicity of faecal water, but increased levels 
of mucin-type glycoproteins were observed in the faecal samples.

As FOS are included in many functional foods and nutritional products for their beneficial health effects, it is important to verify the observations mentioned above in human subjects receiving a regular diet, unrestricted in $\mathrm{Ca}$. We therefore performed a randomised, double-blind, placebo-controlled crossover trial in which the effect of high doses of dietary short-chain FOS on the cytotoxicity of faecal water, on the activity of alkaline phosphatase (ALP) and on the faecal content of $O$-linked oligosaccharides was evaluated in healthy adults who maintained their regular diet.

\section{Materials and methods}

\section{Subjects}

Twelve healthy subjects, six men and six women, aged 18-35 years were eligible for inclusion in the study. None suffered from gastrointestinal disease or had a history of gastrointestinal disease. They did not use any products containing added prebiotic oligosaccharides, probiotic bacteria or added fibre. None of the participants was pregnant or lactating, or had been using antibiotics and/or laxative drugs (for up to 3 months before inclusion).

\section{Study design}

The study was a randomised, double-blind, placebo-controlled crossover trial with two study periods separated by a wash-out period. Healthy adults were recruited via advertisements and posters in the region of Wageningen, The Netherlands. Participation in the study was voluntary, and written informed consent was obtained before the start of the study.

During a pre-trial visit to the participants in their homes, a small questionnaire with questions on the use of nutritional supplements with fibre and/or probiotics was filled out, body weight was assessed, the study diary was explained, the study products were handed out, and a small freezer (LEC Refrigeration, Prescot, UK) was provided for the storage of stools.

Two groups of six adults used two different test products in random order for 2 weeks with a 1-week run-in period included in both periods. The two intervention periods were separated by a wash-out period of 2 weeks. The products were FOS (Raftilose p95, degree of polymerisation 2-7; Orafti, Tienen, Belgium) and maltodextrin (Fantomalt; Nutricia, Zoetermeer, The Netherlands) as a control product, provided in sachets of $5 \mathrm{~g}$ each. A computerised randomisation list was obtained by a logistics manager, independent of the principal researchers. The participants were asked to consume two sachets at breakfast, two sachets at lunch and one or two sachets at dinner. In total, participants used five or six sachets daily, depending on body weight. After a run-in period (day 1 , $5 \mathrm{~g}$; days 2-4, $10 \mathrm{~g}$; days 5-7,20 g), the total daily dose of FOS was $25 \mathrm{~g}$ (five sachets) for subjects with a body weight below $70 \mathrm{~kg}$, and $30 \mathrm{~g}$ (six sachets) for subjects with a body weight over $70 \mathrm{~kg}$. No other dietary changes were prescribed, and the participants were asked to maintain their regular food intake and daily activities.
During the study period, a study diary was filled in with questions on the frequency and consistency of stools, medicine intake and gastrointestinal complaints (constipation, diarrhoea, flatulence, bloating, stomach ache, cramps, nausea, regurgitations, vomiting). The consistency of stools was rated on a five-point scale $(1=$ watery, $2=$ soft, pudding-like, $3=$ soft, formed, $4=$ dry, formed, $5=$ dry, hard pellets), and gastrointestinal complaints were rated on a four-point scale $(1=$ none, $2=$ mild, $3=$ moderate, $4=$ severe $)$. In addition, deviations from regular food intake were recorded in the 'remarks' section of the study diary. At the end of the 2-week intervention periods, the participants were asked to collect their stools on three consecutive days (days 12, 13 and 14, and days 40, 41 and 42). All stools were stored at $-20^{\circ} \mathrm{C}$ until transport to Numico Research. The study was approved by the Medical Ethics Committee of the Radboud University Nijmegen Medical Centre in Nijmegen.

\section{Biochemical analysis of faeces}

Preparation of faecal samples. After storage at $-20^{\circ} \mathrm{C}$, all individual 3-d stool collections were thawed at $4^{\circ} \mathrm{C}$, pooled and homogenised, and the total wet weight was determined. A part of the pooled faeces was freeze dried for further analyses, and the percentage dry weight was determined.

Measurements of $\mathrm{pH}$. Wet faeces were diluted five times in saline, and the $\mathrm{pH}$ was measured directly in faeces at room temperature using a Handylab $\mathrm{pH}$ meter (Schott Glas, Mainz, Germany) equipped with an Inlab 423 pH electrode (Mettler-Toledo, Tiel, The Netherlands).

SCFA and lactate. For the determination of SCFA, wet faeces were thawed in ice water, diluted 10 times (w/v) in milliQ double-distilled water and homogenised for $10 \mathrm{~min}$ using a stomacher (IUL Instruments, Barcelona, Spain). Homogenised faeces $350 \mu \mathrm{l}$ were mixed with $200 \mu \mathrm{l} 5 \%$ (v/v) formic acid, $100 \mu \mathrm{l} 1.25 \mathrm{~g} / \mathrm{l}$ 2-ethylbutyric acid (Sigma-Aldrich, Zwijndrecht, The Netherlands) and $350 \mu \mathrm{l}$ milliQ water. The samples were centrifuged for $5 \mathrm{~min}$ at $16000 \mathrm{~g}$ to remove large particles, and the supernatant was stored at $-20^{\circ} \mathrm{C}$. SCFA (acetic, propionic, $n$ butyric acids) were quantitatively determined as described previously by using gas chromatography with 2-ethylbutyric acid as an internal standard (Knol et al. 2004), and were expressed as $\mu \mathrm{mol} / \mathrm{g}$ wet faeces. For the lactate analyses, faecal samples were diluted two times in PBS (pH 7.4) and homogenised using a stomacher IUL Instruments, Barcelona, Spain. Thereafter, the concentrations of $\mathrm{L}$ - and D-lactate in the samples were determined spectrophotometrically with L- and D-lactic acid enzyme kits (Scil Diagnostics GmbH, Viernheim, Germany). The results for lactate are expressed as $\mu \mathrm{mol} / \mathrm{g}$ wet faeces.

Cytotoxicity of faecal water. Faecal water was prepared by reconstitution of freeze-dried faeces with double-distilled water at a final concentration of $20 \% \mathrm{w} / \mathrm{v}$. After incubation for $1 \mathrm{~h}$ at $37^{\circ} \mathrm{C}$, the samples were centrifuged for $30 \mathrm{~min}$ at $10000 \mathrm{~g}$. Supernatants were then centrifuged for $10 \mathrm{~min}$ at $10000 \mathrm{~g}$. Osmolality was measured to assure physiological ionic strength $(300 \mathrm{mOsmol} / \mathrm{kg})$. The samples were stored at $-20^{\circ} \mathrm{C}$ until analysis.

Cytotoxicity was evaluated as previously described, with minor changes (Govers \& Van der Meer, 1993). Increasing volumes of faecal water were buffered with $100 \mathrm{~mm}$-MOPS (3-N-morpholino-propanesulfonic acid) buffer, $\mathrm{pH} 7 \cdot 4$, to a total volume of 
$80 \mu \mathrm{l}$. After preincubation for $5 \mathrm{~min}$ at $37^{\circ} \mathrm{C}, 20 \mu \mathrm{l}$ of a washed human erythrocyte suspension was added (final haematocrit $5 \%$ ) and incubated for $2 \mathrm{~h}$ at $37^{\circ} \mathrm{C}$. The intact erythrocytes were precipitated by centrifugation for $1 \mathrm{~min}$ and washed twice with $154 \mathrm{~mm}-\mathrm{NaCl}$. After acidification with TCA (final $5 \% \mathrm{w} / \mathrm{v}$ ) and subsequent centrifugation, the $\mathrm{K}$ content of the supernatant was measured by atomic emission spectrophotometry (Atomic Absorption Spectrophotometer 3030; Perkin Elmer, Life and Analytical Sciences Inc., Wellesley, MA 02481-4078, USA). Simultaneously, erythrocytes were incubated in $154 \mathrm{~mm}-\mathrm{NaCl}$ (0\% haemolysis) and in double-distilled water (100\% haemolysis). The percentage haemolysis was calculated from these controls. Cytotoxicity was quantified as the area under the haemolytic curve, and the results were expressed as a percentage of the maximal area, which implies $100 \%$ lysis at each dilution of faeces.

Activity of alkaline phosphatase. Freeze-dried faeces were diluted twenty times in PBS (w/v), homogenised and centrifuged for $5 \mathrm{~min}$ at $1500 \mathrm{~g}$. The supernatants were then collected, and PBS was added (faecal suspension). ALP activity in the supernatants was determined as described earlier, with minor modifications (Lapre et al. 1993; Bovee-Oudenhoven et al. 1996). In brief, $5 \mu \mathrm{l}$ faecal suspension or standard ALP $(0-2.5 \mathrm{mU} / 5 \mu \mathrm{l}$; Sigma-Aldrich) was incubated with $50 \mu l$ substrate ( $p$-nitrophenylphosphate, $6 \mathrm{~mm}$; Sigma-Aldrich) in glycine buffer (glycine (100 mM), MP Biomedicals BV, Zoetermeer, The Netherlands; $\mathrm{MgCl}_{2} \cdot 6 \mathrm{H}_{2} \mathrm{O}$ (1 mM) and $\mathrm{ZnCl}_{2}(1 \mathrm{~mm})$ Merck Eurolab, VWR International, Roden, The Netherlands) during $30 \mathrm{~min}$ incubation at $37^{\circ} \mathrm{C}$. The reaction was stopped with $0.5 \mathrm{ml} \mathrm{NaOH}(0.02 \mathrm{M})$. The absorbance of the $p$-nitrophenol reaction product was determined using a spectrophotometer $(405 \mathrm{~nm}$; Biorad, Veenendaal, The Netherlands). Final dilutions of the faecal suspensions were $1: 150$ and 1:450 to ensure detection in the linear part of the standard curve. The results for ALP activity were expressed as U/g dry faeces.

O-linked oligosaccharides. The excretion of $O$-linked oligosaccharides was determined in the same samples used for the analysis of ALP. Freeze-dried faeces were diluted twenty times in PBS $(\mathrm{w} / \mathrm{v})$, homogenised and centrifuged for $5 \mathrm{~min}$ at $1500 \mathrm{~g}$. The supernatants were then incubated for $10 \mathrm{~min}$ at $95^{\circ} \mathrm{C}$ and $90 \mathrm{~min}$ at $30^{\circ} \mathrm{C}$, and then centrifuged for $1 \mathrm{~min}$ at $15000 \mathrm{~g}$. The supernatants were further diluted to 1:200, 1:500 and 1:1000 suspension. The amount of $O$-linked oligosaccharides in the faecal suspensions was determined as described elsewhere, with minor modifications (Crowther \& Wetmore, 1987; Bovee-Oudenhoven et al. 1996). In brief, $120 \mu \mathrm{l}$ substrate ( $2 \mathrm{ml} 0.6 \mathrm{M}$-2-cyanoacetamide; Sigma-Aldrich) in $10 \mathrm{ml} \mathrm{NaOH} \mathrm{(15} \mathrm{mM;} \mathrm{JTBaker;} \mathrm{Mal-}$ linckrodt Baker BV, Deventer, The Netherlands) was mixed with $100 \mu \mathrm{l}$ faecal suspension or standard $O$-linked glycoprotein $(0-0.3 \mathrm{mg} / \mathrm{ml}, \mathrm{N}$-acetylgalactosamine; Sigma-Aldrich) and incubated for $30 \mathrm{~min}$ at $100^{\circ} \mathrm{C}$. At room temperature, $1 \mathrm{ml}$ borate buffer $(0.6 \mathrm{M}$; Merck) was added and the fluorescence signal measured (FLUOstar Galaxy; BMG Labtechnologies, GmbH, Offenburg, Germany) at excitation wavelength $380 \mathrm{~nm}$ and emission $460 \mathrm{~nm}$. The results for the amounts of $O$-linked oligosaccharides in the faeces are expressed in $\mathrm{mg} / \mathrm{g}$ dry faeces.

\section{Statistical analysis}

The study was a randomised, double-blind, placebo-controlled crossover trial, with two treatment periods involving two groups of six participants in each period. A power calculation indicated that a sample size of twelve healthy adults was necessary to detect a statistically significant difference in faecal water cytotoxicity of $25 \%$, assuming a standard deviation of $22 \%$, with a power of 0.8 and a two-sided significance level of 0.05 (Rafter et al. 1987).

All data were analysed with an ANOVA model with the product (control or FOS), the period (first or second) and the product sequence (FOS then control, or control then FOS) as fixed factors, and subjects as a nested factor (Jones \& Kenward, 2003). Normality was checked with the Shapiro-Wilk test of normality on the residuals. Means of the questionnaire data on stool characteristics and gastrointestinal complaints were calculated for the second week of intervention. The data from the run-in period were not included in the analysis. There appeared to be no significant carry-over or period effects, and therefore the data from the treatments in the two periods were pooled. The results of the product periods are expressed as means with their standard errors, and the $95 \%$ CI of the difference. All statistical analyses have been performed with SPSS version 12.0.1 (SPSS Inc., Chicago, IL, USA). Differences between the product groups were considered statistically significant at $P<0.05$.

\section{Results}

All twelve participants (six men, six women) completed the study. The mean age at enrolment was 21.4 (SD 2.0) years, and mean weight and height were $66.4 \mathrm{~kg}$ (SD $8.3 \mathrm{~kg}$ ) and $1.79 \mathrm{~m}(\mathrm{SD} 0.08 \mathrm{~m})$ respectively. One stool collection was missing in the analysis owing to the unexpected absence of the participant during the time of the sampling. Hence, the results of eleven participants are presented. Compliance, determined by counting the number of unused sachets at the end of the study period, was high $(98.4 \%)$, indicating that the prescribed daily dose $(25-30 \mathrm{~g})$ was achieved throughout the study periods.

\section{Stool characteristics and gastrointestinal symptoms}

Stool results are shown in Table 1. Stool frequency was significantly higher in the FOS period compared with the control period, with a mean stool frequency of 1.2 (SEM 0.06) per day in the control period and 1.5 (SEM 0.06) per day in the FOS period $(P=0 \cdot 014)$. Total stool output tended to be higher in the FOS period $(\mathrm{F}(1,9)=3 \cdot 4, P=0.097)$, and stool consistency was similar in both periods $(\mathrm{F}(1,10)=0 \cdot 3$, $P=0 \cdot 607)$.

In the control group, mild gastrointestinal complaints were recorded thirty-one times, and moderate complaints were recorded twice (stomach ache once, flatulence once). Severe complaints were not recorded. In the FOS group, mild gastrointestinal complaints were reported twenty-nine times, and moderate complaints were reported twenty-four times (diarrhoea four times (one subject $3 \mathrm{~d}$, one subject $1 \mathrm{~d}$ ), stomach ache twice (one subject $2 \mathrm{~d}$ ), bloating three times (three subjects $1 \mathrm{~d}$ ), flatulence ten times (one subject $3 \mathrm{~d}$, two subjects $2 \mathrm{~d}$, three subjects $1 \mathrm{~d}$ ), nausea once, and cramps four times (two subjects $2 \mathrm{~d}$ )). Severe complaints were reported eleven times (diarrhoea once, stomach ache twice (two subjects $1 \mathrm{~d}$ ), bloating once, flatulence seven 
Table 1. Effects of dietary fructo-oligosaccharides (FOS) and maltodextrin (control) on stool characteristics in healthy adults ( $n 11$ for stool output and percentage dry weight, $n 12$ for stool frequency and stool consistency)

\begin{tabular}{|c|c|c|c|c|c|}
\hline & \multicolumn{2}{|c|}{ Control period } & \multicolumn{2}{|c|}{ FOS period } & \multirow[b]{2}{*}{$95 \% \mathrm{Cl}$ of the difference $(\mathrm{F}, P)$} \\
\hline & Mean & SEM & Mean & SEM & \\
\hline \multicolumn{6}{|l|}{ Stool output } \\
\hline Wet faeces $(g / d)$ & 174 & $19 \cdot 3$ & 225 & $19 \cdot 3$ & -112.3 to $11 \cdot 2(F(1,9)=3 \cdot 4, P=0.097)$ \\
\hline Dry faeces $(\mathrm{g} / \mathrm{d})$ & $46 \cdot 6$ & $4 \cdot 1$ & $52 \cdot 1$ & $4 \cdot 1$ & -18.7 to $7.7(F(1,9)=0.9, P 0.369)$ \\
\hline Percentage dry weight & $26 \cdot 2$ & $1 \cdot 0$ & 23.9 & $1 \cdot 0$ & -0.9 to $5.5(\mathrm{~F}(1,9)=2.7, P 0.135)$ \\
\hline Stool frequency (mean/d) & $1 \cdot 2$ & 0.06 & 1.5 & 0.06 & -0.4 to $-0.1(F(1,10)=8.9, P=0.014)$ \\
\hline Stool consistency (mean/d) & $3 \cdot 2$ & 0.09 & $3 \cdot 1$ & 0.09 & -0.2 to $0.3(F(1,10)=0.3, P=0.607)$ \\
\hline
\end{tabular}

times (one subject $1 \mathrm{~d}$, one subject $6 \mathrm{~d}$ ), and cramps once). No significant differences between the control and the FOS period were observed in the mean occurrence of these complaints, except for flatulence: in the FOS period, participants recorded a significantly higher score of flatulence compared with the control period (with a mean score of 1.8 (SEM 0.18) in the FOS period and 1.2 (SEM $0 \cdot 18)$ in the control period; $\mathrm{F}(1,10)=5 \cdot 6, P=0 \cdot 039)$.

\section{pH, SCFA and lactate in stools}

There was a trend towards a lower stool $\mathrm{pH}$ in the FOS period compared with the control period $(\mathrm{F}(1,9)=4 \cdot 4, P=0.065)$. The proportions of SCFA changed owing to FOS supplementation, with higher proportions of acetate $(\mathrm{F}(1,9)=11.2$, $P=0.009)$ and lower proportions of butyrate $(\mathrm{F}(1,9)=17 \cdot 1$, $P=0 \cdot 003)$. No major differences were observed in the concentration of total SCFA in the faeces. Faecal lactate concentrations were higher in the FOS group, but the difference was not statistically significant $(\mathrm{F}(1,9)=0 \cdot 7, P=0 \cdot 376)$. The results are shown in Table 2.

Cytotoxicity of faecal water, alkaline phosphatase activity and faecal contents of O-linked oligosaccharides

FOS tended to reduce the cytotoxicity of faecal water, with mean cytotoxicity levels of 37.5 (SEM 6.9) \% and 18.5 (SEM 6.9) $\%$ in the control period and the FOS period, respectively $(\mathrm{F}(1,9)=3 \cdot 8, P=0 \cdot 084$; Fig. 1). No difference was observed in mean faecal ALP activity in the control treatment period (27.7 (SEM 2.9) U/g dry faeces) and the FOS treatment period (24.6 (SEM 3.2) U/g dry faeces; $F(1,9)=0.5$, $P=0.496$; Fig. 2). In the same faecal samples, the amount of $O$-linked oligosaccharide in the faeces was determined to estimate faecal mucin content (Fig. 3). The mean faecal excretion of $O$-linked oligosaccharides in the control and the FOS periods was 49.9 (SEM 4.0) and 53.5 (SEM 4.3) mg/g dry faeces, respectively. No statistical significant difference was observed $(\mathrm{F}(1,9)=0 \cdot 4, P=0.553)$.

\section{Discussion}

Our results demonstrate that the cytotoxicity of faecal water, faecal ALP activity and the faecal concentration of mucintype oligosaccharides were not affected by the intake of dietary FOS in healthy adults with a regular diet unrestricted in Ca.

FOS were provided for 2 weeks, with a run-in of 1 week in each period. The dose of FOS used in the current study was $25-30 \mathrm{~g} / \mathrm{d}$, which in Europe is far above the estimated mean daily intake of FOS and inulin from natural sources, namely $3 \cdot 2-11 \cdot 3 \mathrm{~g}$, with peak values of up to $20 \mathrm{~g}$ (Van Loo, 1995). FOS are resistant to hydrolysis by human small intestinal enzymes and enter the colon intact. In the colon, FOS are fermented by intestinal bacteria, and several studies have demonstrated an increase in the intestinal bifidobacterium count within a few weeks of supplementation with FOS (Gibson et al. 1995; Bouhnik et al. 1996, 1999; Buddington et al. 1996; Kleessen et al. 1997; Tuohy et al. 2001). End products of fermentation by bifidobacteria include lactate, SCFA (in particular acetate) and gases such as $\mathrm{CO}_{2}$ and $\mathrm{H}$. An analysis of these end products in faeces gives an indication of bacterial fermentation.

In our study, FOS affected bacterial fermentation, as observed by a trend towards a lower $\mathrm{pH}$, a statistically significant higher proportion of acetate and a tendency towards higher levels of lactate. Furthermore, FOS intake resulted in a more frequently reported occurrence of flatulence, a higher stool frequency and a trend towards a higher stool output, all

Table 2. Effects of dietary fructo-oligosaccharides (FOS) and maltodextrin (control) on pH, SCFA and lactate in the faeces of healthy adults ( $n 11)$

\begin{tabular}{|c|c|c|c|c|c|}
\hline & \multicolumn{2}{|c|}{ Control period } & \multicolumn{2}{|c|}{ FOS period } & \multirow[b]{2}{*}{$95 \% \mathrm{Cl}$ of the difference $(\mathrm{F}, P)$} \\
\hline & Mean & SEM & Mean & SEM & \\
\hline $\mathrm{pH}$ & $6 \cdot 6$ & 0.16 & $6 \cdot 1$ & 0.18 & -0.0 to $1.0(F(1,9)=4.4, P=0.065)$ \\
\hline Lactate ( $\mu \mathrm{mol} / \mathrm{g}$ wet faeces) & $5 \cdot 6$ & 1.7 & $8 \cdot 0$ & $2 \cdot 0$ & -8.6 to $3.6(F(1,9)=0.7, P=0.376)$ \\
\hline Total SCFA ( $\mu \mathrm{mol} / \mathrm{g}$ wet faeces) & $97 \cdot 3$ & $6 \cdot 7$ & $90 \cdot 6$ & $7 \cdot 4$ & -15.8 to $29.3(\mathrm{~F}(1,9)=0.5, P=0.517)$ \\
\hline Acetate ( $\%$ of total SCFA) & $62 \cdot 0$ & $2 \cdot 0$ & 72 & $2 \cdot 2$ & -16.9 to $-3.3(\mathrm{~F}(1,9)=11.2, P=0.009)$ \\
\hline Propionate ( $\%$ of total SCFA) & $17 \cdot 5$ & $1 \cdot 3$ & 14.9 & 1.4 & -1.9 to $7.0(F(1,9)=1.7, P=0.224)$ \\
\hline Butyrate (\% of total SCFA) & $20 \cdot 5$ & $1 \cdot 2$ & $13 \cdot 0$ & 1.4 & 3.4 to $11.7(\mathrm{~F}(1,9)=17 \cdot 1, P=0.003)$ \\
\hline
\end{tabular}




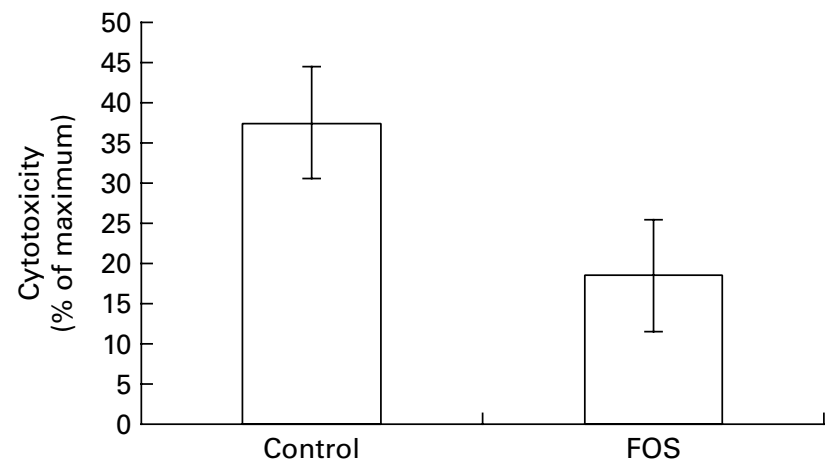

Fig. 1. Cytotoxicity of faecal water. The bars represent the percentage cytotoxicity, expressed as the percentage and standard error of the maximum area under the curve (implying $100 \%$ lysis) in the control group ( $n 11)$ and the fructo-oligosaccharide (FOS) group ( $n$ 11). The difference between the two groups was not statistically significant $(F(1,9)=3 \cdot 8, P=0.084,95 \% \mathrm{Cl}$ of the difference -3.12 to $41.3 \%$ ).

known to occur after the intake of high doses of non-digestible fibres such as FOS (Gibson et al. 1995; Bouhnik et al. 1996; Buddington et al. 1996; Kleessen et al. 1997; Tuohy et al. 2001). Serious adverse events were not reported.

The daily intake of FOS did not affect intestinal epitheliolysis, as indicated by the cytotoxicity of faecal water and the faecal activity of ALP. The cytotoxicity of faecal water is determined by the presence of potential luminal toxic compounds that can harm the intestinal epithelial membrane. ALP is an enzyme that is located within the apical membrane of enterocytes. Damage to the apical membrane or epithelial shedding may result in the release of ALP into the faecal stream, hence ALP can be used as a marker for intestinal epitheliolysis (McNeil \& Ito, 1989; Lapre et al. 1991; Bovee-Oudenhoven et al. 1996). Since lysis is determined by the cytotoxicity of luminal contents as well as by the susceptibility of the plasma membrane exposed to these surfactants, a combination of both analyses is relevant for the quantification of epitheliolysis. It was revealed that both the cytotoxicity of faecal water and the activity of ALP in faecal water were not affected by the intake of FOS. Moreover, the cytotoxicity of faecal water tended to be lower in

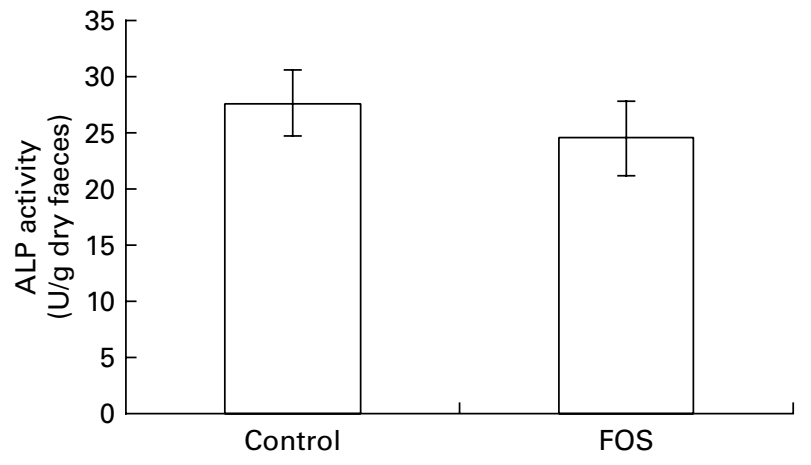

Fig. 2. Alkaline phosphatase (ALP) activity in the faeces, a marker for epithelial turnover and shedding. The bars represent the means with their standard errors of ALP activity in U/g dry faeces with in the control group ( $n 11)$ and the fructo-oligosaccharides (FOS) group ( $n$ 11). There was no statistica significant difference between the two groups $(F(1,9)=0.5, P=0.496,95 \%$ $\mathrm{Cl}$ of the difference -6.8 to $13.0 \mathrm{U} / \mathrm{g}$ dry faeces).

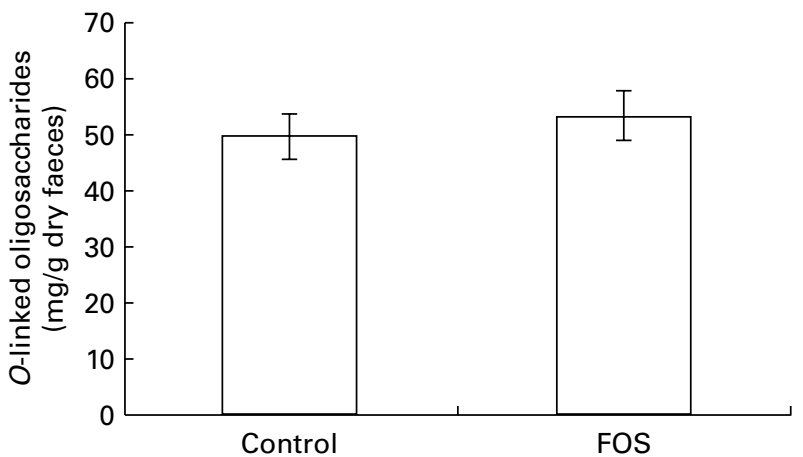

Fig. 3. O-linked oligosaccharide concentration in the faeces to estimate the amount of mucin-type glycoprotein. The bars represent the means with their standard errors of the amounts of $O$-linked oligosaccharides in $\mathrm{mg} / \mathrm{g}$ dry faeces in the control group ( $n$ 11) and the fructo-oligosaccharides (FOS) group ( $n$ 11). There was no significant difference between the two groups $(\mathrm{F}(1,9)=0.4, P=0.553,95 \% \mathrm{Cl}$ of the difference -17.0 to $9.7 \mathrm{mg} / \mathrm{g}$ dry faeces).

the FOS period compared with the control period, with a mean observed difference of $19.1 \%(P=0.084)$, indicating a protective effect of FOS against cytotoxic components in the colon.

The susceptibility of the intestinal epithelial membrane to toxic compounds is also determined by the intestinal mucus gel layer covering the intestinal epithelium. The mucus gel layer consists of mucins, which are glycoproteins produced and secreted by goblet cells in the gastrointestinal tract. Many physiological or pathogenic stimuli enhance mucin secretion, which is thought to contribute to the intestinal barrier function, protecting the gastrointestinal mucosa from mechanical, chemical and microbial challenge (Strous \& Dekker, 1992). For example, bacterial fermentation products such as acetate are known to support mucoprotection by increasing intestinal mucin expression and secretion (Finnie et al. 1995; Sakata \& Setoyama, 1995; Shimotoyodome et al. 2000; Willemsen et al. 2003).

On the other hand, changes in the excretion of mucin-type oligosaccharides may also indicate irritation or challenge of the intestinal epithelium. Mucins consist of a protein core, with $O$-linked oligosaccharide side chains (Strous \& Dekker, 1992; Deplancke \& Gaskins, 2001). Thus, analysis of $O$-linked carbohydrates in the faeces gives an estimate of the amount of mucin glycoprotein secreted (Bovee-Oudenhoven et al. 1996). Under normal physiological conditions, mucins can only be detected at low concentrations in the faeces, since both commensal and pathogenic intestinal bacteria are able to ferment shedded mucins (Hoskins, 1981; Hoskins et al. 1985; Deplancke \& Gaskins, 2001). Hence, the actual amount of mucin in the faeces is a result of the production, shedding and fermentation of mucins in the gastrointestinal tract.

In our human volunteer study, no significant differences were observed in the excretion of mucin-type oligosaccharides in the faeces between the FOS period and the control period. These results are in agreement with results on the cytotoxicity of faecal water and the activity of ALP. The analysis of mucins in faeces is troublesome, and although the $O$-glycosylation methods may give an indication of intestinal mucin excretion, such assays are not reliable for estimating intestinal 
cell damage. The excretion of mucin in healthy adults was previously studied by Ouwehand et al. (2000), using a colorimetric assay. In this study, the excretion of mucin did not differ between adults consuming a high-fibre diet and those consuming a low-fibre diet. The mean faecal concentration was approximately $6 \mathrm{mg} / \mathrm{g}$ wet faeces, which corresponds to a concentration of approximately $24 \mathrm{mg} / \mathrm{g}$ dry faeces. In another study, an increased faecal concentration of mucintype oligosaccharides was observed in healthy males receiving a diet with $20 \mathrm{~g}$ of FOS daily for 2 weeks, in combination with a diet restricted in Ca (Ten Bruggencate et al. 2004b).

Our results differ from the results of other studies, in which a higher cytotoxicity of faecal water and an increased excretion of mucin-type oligosaccharides were observed in Ca-restricted rats fed high doses of FOS (Bovee-Oudenhoven et al. 2003; Ten Bruggencate et al. 2003, 2004a), and a higher excretion of mucin-type oligosaccharides was observed in humans receiving $20 \mathrm{~g}$ of FOS in combination with a diet restricted in Ca (Ten Bruggencate et al. 2004b). As another recent study by the same group (Ten Bruggencate et al. 2004a) demonstrated that $\mathrm{Ca}$ inhibited the irritative effects of FOS in rats, the dietary $\mathrm{Ca}$ restriction in the studies mentioned above may have had an adverse effect on the cytotoxicity and/or mucin excretion. In the small intestine, $\mathrm{Ca}$ and $\mathrm{PO}_{4}$ form an insoluble complex, which can dissolve in the more acidic environment of the colon. Hence, $\mathrm{Ca}$ is known to increase the buffering capacity of the intestinal lumen (Govers \& Van der Meer, 1993; Govers et al. 1996), and to bind cytotoxic components, precipitating in the lumen, thereby decreasing the cytotoxic activity in the colon (Govers et al. 1994).

As the present study was performed under habitual dietary conditions, without any dietary $\mathrm{Ca}$ restriction, we conclude that dietary FOS in a dose of up to $25-30 \mathrm{~g} / \mathrm{d}$, in combination with a regular diet unrestricted in $\mathrm{Ca}$, do not affect the cytotoxicity of faecal water, faecal ALP activity or the faecal concentration of mucin-type oligosaccharides in human adults.

\section{Acknowledgements}

The authors would like to thank the volunteers (Wageningen, The Netherlands) who participated in the study, Madelon Brummelhuis (Wageningen University) for the practical execution of the study, Jürgen Jelinek (Friedrichsdorf, Germany) and Rob Verdooren (Wageningen, The Netherlands) for statistical advice, Günther Raffler of the Central Laboratories Friedrichsdorf (Friedrichsdorf, Germany) for the $\mathrm{K}$ analyses, and Roy Beernink for the ALP and $O$-glycosylation analyses (Wageningen, The Netherlands). Furthermore, we would like to thank the investigators of NIZO Food Research (Sandra ten Bruggencate, Ingeborg Bovee, Roelof Van der Meer) for the stimulating discussion regarding their own and the present study.

\section{References}

Bouhnik Y, Flourie B, Riottot M, Bisetti N, Gailing MF, Guibert A, Bornet F \& Rambaud JC (1996) Effects of fructo-oligosaccharides ingestion on fecal bifidobacteria and selected metabolic indexes of colon carcinogenesis in healthy humans. Nutr Cancer 26, 21-29.
Bouhnik Y, Vahedi K, Achour L, Attar A, Salfati J, Pochart P, Marteau P, Flourie B, Bornet F \& Rambaud JC (1999) Short-chain fructo-oligosaccharide administration dose-dependently increases fecal bifidobacteria in healthy humans. J Nutr 129, 113-116.

Bovee-Oudenhoven IM, ten Bruggencate SJ, Lettink-Wissink ML \& van der Meer R (2003) Dietary fructo-oligosaccharides and lactulose inhibit intestinal colonisation but stimulate translocation of salmonella in rats. Gut 52, 1572-1578.

Bovee-Oudenhoven I, Termont D, Dekker R \& Van der Meer R (1996) Calcium in milk and fermentation by yoghurt bacteria increase the resistance of rats to Salmonella infection. Gut 38, 59-65.

Buddington RK, Williams CH, Chen SC \& Witherly SA (1996) Dietary supplement of neosugar alters the fecal flora and decreases activities of some reductive enzymes in human subjects. Am J Clin Nutr 63, 709-716.

Crowther RS \& Wetmore RF (1987) Fluorometric assay of O-linked glycoproteins by reaction with 2-cyanoacetamide. Anal Biochem 163, 170-174.

Deplancke B \& Gaskins HR (2001) Microbial modulation of innate defense: goblet cells and the intestinal mucus layer. Am J Clin Nutr 73, S1131-S1141.

Finnie IA, Dwarakanath AD, Taylor BA \& Rhodes JM (1995) Colonic mucin synthesis is increased by sodium butyrate. Gut 36, 93-99.

Gibson GR, Beatty ER, Wang X \& Cummings JH (1995) Selective stimulation of bifidobacteria in the human colon by oligofructose and inulin. Gastroenterology 108, 975-982.

Govers MJ, Termont DS, Lapre JA, Kleibeuker JH, Vonk RJ \& Van der Meer R (1996) Calcium in milk products precipitates intestinal fatty acids and secondary bile acids and thus inhibits colonic cytotoxicity in humans. Cancer Res 56, $3270-3275$.

Govers MJ, Termont DS \& Van der Meer R (1994) Mechanism of the antiproliferative effect of milk mineral and other calcium supplements on colonic epithelium. Cancer Res 54, 95-100.

Govers MJ \& Van der Meer R (1993) Effects of dietary calcium and phosphate on the intestinal interactions between calcium, phosphate, fatty acids, and bile acids. Gut 34, 365-370.

Hoskins LC (1981) Human enteric population ecology and degradation of gut mucins. Dig Dis Sci 26, 769-772.

Hoskins LC, Agustines M, McKee WB, Boulding ET, Kriaris M \& Niedermeyer G (1985) Mucin degradation in human colon ecosystems. Isolation and properties of fecal strains that degrade $\mathrm{ABH}$ blood group antigens and oligosaccharides from mucin glycoproteins. J Clin Invest 75, 944-953.

Jones B \& Kenward M (2003) Design and Analysis of Cross-over Trials, 2nd ed., Boca Raton, FL: Chapman \& Hall/CRC.

Kleessen B, Sykura B, Zunft HJ \& Blaut M (1997) Effects of inulin and lactose on fecal microflora, microbial activity, and bowel habit in elderly constipated persons. Am J Clin Nutr 65, $1397-1402$.

Knol J, Scholtens PAMJ, Kafka C, et al. (2005) Colon microflora in infants fed formula with galacto- and fructo-oligosaccharides: more like breast-fed infants. J Pediatr Gastroenterol Nutr 40, 36-42.

Kolida S, Tuohy K \& Gibson GR (2002) Prebiotic effects of inulin and oligofructose. Br J Nutr 87, S193-S197.

Lapre JA, De Vries HT, Koeman JH \& Van der Meer R (1993) The antiproliferative effect of dietary calcium on colonic epithelium is mediated by luminal surfactants and dependent on the type of dietary fat. Cancer Res 53, 784-789.

Lapre JA, Kleibeuker J \& van der Meer R (1991) Intestinal alkaline phosphatase in fecal water reflects epitheliolysis and is decreased by dietary calcium. Gastroenterology 100, A378.

McNeil PL \& Ito S (1989) Gastrointestinal cell plasma membrane wounding and resealing in vivo. Gastroenterology 96, 1238-1248.

Ouwehand AC, Grasten S, Niemi P, Mykkanen H \& Salminen S (2000) Wheat or rye supplemented diets do not affect faecal 
mucus concentration or the adhesion of probiotic micro-organisms to faecal mucus. Lett Appl Microbiol 31, 30-33.

Rafter JJ, Child P, Anderson AM, Alder R, Eng V \& Bruce WR (1987) Cellular toxicity of fecal water depends on diet. Am J Clin Nutr 45, 559-563.

Sakata T \& Setoyama H (1995) Local stimulatory effect of short-chain fatty acids on the mucus release from the hindgut mucosa of rats (Rattus norvegicus). Comp Biochem Physiol A Physiol 111, 429-432.

Shimotoyodome A, Meguro S, Hase T, Tokimitsu I \& Sakata T (2000) Short chain fatty acids but not lactate or succinate stimulate mucus release in the rat colon. Comp Biochem Physiol A Mol Integr Physiol 125, 525-531.

Strous GJ \& Dekker J (1992) Mucin-type glycoproteins. Crit Rev Biochem Mol Biol 27, 57-92.

Ten Bruggencate SJ, Bovee-Oudenhoven IM, Lettink-Wissink ML, Katan MB \& Van Der Meer R (2004a) Dietary fructo-oligosaccharides and inulin decrease resistance of rats to salmonella: protective role of calcium. Gut 53, 530-535.
Ten Bruggencate SJM, Bovee-Oudenhoven IM, Lettink-Wissink ML, Katan MB \& van der meer R (2004b) Dietary fructo-oligosaccharides and the intestinal barrier in humans. PhD Thesis, Wageningen University, The Netherlands.

Ten Bruggencate SJ, Bovee-Oudenhoven IM, Lettink-Wissink ML \& Van der Meer R (2003) Dietary fructo-oligosaccharides dosedependently increase translocation of salmonella in rats. $J$ Nutr 133, 2313-2318.

Tuohy KM, Kolida S, Lustenberger AM \& Gibson GR (2001) The prebiotic effects of biscuits containing partially hydrolysed guar gum and fructo-oligosaccharides - a human volunteer study. $\mathrm{Br} J$ Nutr 86, 341-348.

Van Loo J (1995) On the presence of inulin and oligofructose as natural ingredients in the Western diet. Crit Rev Food Sci Nutr 36, $525-552$.

Willemsen LE, Koetsier MA, van Deventer SJ \& van Tol EA (2003) Short chain fatty acids stimulate epithelial mucin 2 expression through differential effects on prostaglandin $\mathrm{E}(1)$ and $\mathrm{E}(2)$ production by intestinal myofibroblasts. Gut 52, 1442-1447. 